University of Nebraska - Lincoln

DigitalCommons@University of Nebraska - Lincoln

May 1986

\title{
Crystal structure and charge transfer energies of complexes of the donor biphenylene with the acceptors TCNB and PMDA
}

John J. Stezowski

University of Nebraska - Lincoln, jjs@unlserve.unl.edu

Rolf-Dietrich Stigler

Universität Stuttgart

Norbert Karl

Universität Stuttgart

Follow this and additional works at: https://digitalcommons.unl.edu/chemistrystezowski

Part of the Chemistry Commons

Stezowski, John J.; Stigler, Rolf-Dietrich ; and Karl, Norbert, "Crystal structure and charge transfer energies of complexes of the donor biphenylene with the acceptors TCNB and PMDA" (1986). John J. Stezowski Publications. 4.

https://digitalcommons.unl.edu/chemistrystezowski/4

This Article is brought to you for free and open access by the Published Research - Department of Chemistry at DigitalCommons@University of Nebraska - Lincoln. It has been accepted for inclusion in John J. Stezowski Publications by an authorized administrator of DigitalCommons@University of Nebraska - Lincoln. 


\title{
Crystal structure and charge transfer energies of complexes of the donor biphenylene with the acceptors TCNB and PMDA ${ }^{\text {a) }}$
}

\author{
John J. Stezowski, Rolf-Dietrich Stigler, and Norbert Karl \\ Institut für Organische Chemie, Biochemie und Isotopenforschung und Physikalisches Institut, Teilinstitut 3 \\ der Universität Stuttgart, D-7000 Stuttgart 80, Federal Republic of Germany
}

(Received 2 August 1985; accepted 26 November 1985)

Biphenylene:tetracyanobenzene ( $B P N$ :TCNB), $\mathrm{C}_{12} \mathrm{H}_{8}: \mathrm{C}_{10} \mathrm{H}_{2} \mathrm{~N}_{4}$, crystallizes in a monoclinic lattice space group $P 2{ }_{1} / a, Z=2$, with $a=15.215(2) \AA, b=7.260$ (1) $\AA, c=7.573$ (1) $\AA$, $\beta=90.05(1)^{\circ}$ at $296 \mathrm{~K}$. The BPN donor and TCNB acceptor molecules form two symmetryrelated mixed linear [001] stacks with the donor and acceptor molecular planes tilted to each other (within the individual stacks) at an angle of $\Phi=7.6^{\circ}$. Biphenylene:pyromelliticdianhydride (BPN:PMDA), $\mathrm{C}_{12} \mathrm{H}_{8}: \mathrm{C}_{10} \mathrm{H}_{2} \mathrm{O}_{6}$, crystallizes in two different monoclinic modifications, both space group $P 2_{1} / a, Z=2$, one red, and the other black. The red form (stable above $\sim 400 \mathrm{~K}$ ) with $a=9.280(1) \AA, b=11.869(2) \AA, c=7.293(1) \AA, \beta=98.68(2)^{\circ}$ at $\sim 120$ $\mathrm{K}$, exhibits mixed linear stacks $\left(\Phi=4.1^{\circ}\right)$. The lattice parameters of the black form (stable below $\sim 400 \mathrm{~K}$ ) are $a=13.368(1) \AA, b=5.809$ (1) $\AA, c=10.443(1) \AA, \beta=102.30(1)^{\circ}$ at $\sim 120 \mathrm{~K}$, and $a=13.450(2) \AA, b=5.917(1) \AA, c=10.464(2) \AA, \beta=101.82(2)^{\circ}$ at $296 \mathrm{~K}$. Its structure also can be viewed as a mixed linear stacking $\left(\Phi=9.9^{\circ}\right)$, but it displays exceptionally little intrastack molecular overlap. Instead, considerable two-dimensional interactions among the donors and among the acceptors can be concluded. Single crystals of all three complexes, obtained by the plate sublimation technique, were optically characterized by their charge transfer absorption edges. These are analyzed in terms of the molecular parameters electron affinity and ionization energy. For BPN:TCNB an intrastack and an interstack CT-absorption edge can be distinguished.

\section{INTRODUCTION}

There are a number of reports in the literature on donor-acceptor complexes between the donors naphthalene $(\mathrm{N})$ and anthracene (A), and the acceptors 1,2,4,5-tetracyanobenzene (TCNB) and pyromellitic-dianhydride (PMDA). Some of these complexes, e.g., N:TCNB, ${ }^{1,2}$ N:PMDA (orange form), ${ }^{3,4}$ and A:TCNB, ${ }^{5,6}$ exhibit either static or dynamic disorder. In an attempt to gain more insight into the underlying packing principles which discriminate static or dynamic disorder from order, we considered it appropriate to use a donor of intermediate size, with the same molecular symmetry and similar molecular properties as naphthalene and anthracene; biphenylene (BPN) was selected. The growth of crystals of the complexes BPN:TCNB, red BPN:PMDA, and black BPN:PMDA, their characterization by differential scanning calorimetry (DSC) and optical charge transfer absorption, and by crystal structure determination are presented here. It will be shown that the crystal structures of BPN:TCNB and black BPN:PMDA reveal mixed linear stacks with considerable nonparallelism of the donor and acceptor molecular planes leading to twodimensional interactions among the donor and among the acceptor molecules, whereas red BPN:PMDA (stable above $\sim 400 \mathrm{~K}$ ) crystallizes in the usual one-dimensional mixed linear stacks. Additional interesting theoretical questions on the nature of the charge transfer interaction arise from the observation of a considerable bathochromic shift of the charge transfer absorption edge, associated with the

\footnotetext{
a) The results were presented in part at the 7 th International Conference on Crystal Growth in Stuttgart, Sept. 1983.
}

BPN:PMDA phase transition from the high temperature red to the low temperature black modification.

\section{EXPERIMENTAL}

Biphenylene (purchased from ICN Pharmaceuticals, N.Y.) was zone refined in a micro zone refiner. ${ }^{7}$ Zone refining of PMDA (from Fluka AG, Buchs, Switzerland) was possible after dehydration of residual pyromellitic acid by slowly heating the material to its melting point under continuous pumping. ${ }^{8}$ TCNB was synthetized from pyromellitic acid via the diimide and tetraamide, recrystallized from ethanol and vacuum sublimed. ${ }^{9}$ Crystals of the complexes were obtained by the plate sublimation technique ${ }^{10}$ at a temperature of $385 \mathrm{~K}(\Delta T=5 \mathrm{~K})$ for BPN:TCNB, at $387 \mathrm{~K}$ $(\Delta T=1 \mathrm{~K})$ for black BPN:PMDA, and at $430 \mathrm{~K}(\Delta T=1$ $\mathrm{K}$ ) for red BPN:PMDA. A stoichiometric (1:1) mixture of the starting materials $(\sim 100 \mathrm{mg})$ was sealed in evacuated sublimation ampoules for this purpose. Melting and phase transition points were measured with a differential scanning calorimeter (Perkin-Elmer, DSC-2).

Crystals, thin enough for optical absorption measurements, could only be obtained in small size. Absorption spectra were therefore measured at fixed wavelength increments through a polarization microscope (Leitz, Ortholux-Pol), equipped with an ocular GW $6.3 \times$, and a $45 \times$ or an APO$25 \times$ objective lens with numerical aperture 0.65 . The illumination aperture was reduced to give a somewhat more parallel light beam. A stabilized tungsten lamp was used, with the IR radiation filtered off by a heat filter (Schott, KG1, 3 $\mathrm{mm}$ ). Light emerging from the ocular was focused on the 
entrance slit of a $25 \mathrm{~cm}$ monochromator (Bausch \& Lomb) and detected at a spectral resolution of $11 \mathrm{~nm}$ by a $10 \mathrm{~mm}^{2}$ silicon photodiode with integral amplifier (Bell and Howell, type $509, \mathrm{NEP} \approx 10^{-12} \mathrm{~W} / \sqrt{\mathrm{Hz}}$ ). For each crystal platelet the transmitted light was measured for two principal polarizations, corrected for reflection losses, and normalized to the intensity in absence of the crystal. The crystal thickness was determined under the microscope with a calibrated micrometer eye piece with the respective crystal platelet placed vertical, and from the vertical shift of the microscope table necessary for focusing either the upper crystal face or the glass support.

Crystals appropriate for $\mathrm{x}$-ray structure analysis were selected and sealed in thin-walled glass capillaries. Diffraction data were collected on a SYNTEX P̄̄ autodiffractometer, equipped with a SYNTEX LT-1 low temperature device. Monochromatized $\mathrm{MoK}_{\alpha}$ radiation ( $\bar{\lambda}=0.71069 \AA$, weighted average between $K_{\alpha 1}$ and $K_{\alpha 2}$ ) was used. Lattice parameters were refined ${ }^{11}$ with automatically centered $2 \theta$ values. Intensities were measured for all reflections with an $\omega$-scan technique.

\section{RESULTS}

\section{Crystals, phase transitions}

BPN:TCNB formed orange single crystals which were partly tabular, partly needle shaped, m.p. $507 \mathrm{~K}$. No phase transition was observed down to $\sim 100 \mathrm{~K}$ (by DSC).

The red modification of BPN:PMDA was obtained above $395.5 \mathrm{~K}$ as isometric single crystals. Incongruent melting begins at $462-464 \mathrm{~K}$. After cooling down to room temperature these crystals transformed slowly (within days or weeks) to a black polycrystalline modification. Single crystals of this modification could be obtained directly by plate sublimation below the phase transition temperature. In contrast to the matt rough surface of transformed material, they characteristically displayed a number of shiny homogeneously reflecting faces. Under the microscope thin platelets appeared dark red in transmission. DSC measurements demonstrated that the phase transition displays hysteresis; on heating it was observed in the interval 399-402 K.

\section{Crystal structures}

All initial models were determined by direct methods, BPN:TCNB and BPN:PMDA (black form), using the X-RAY system, ${ }^{11}$ the red form of BPN:PMDA using MULTAN. ${ }^{12}$ The models were developed by Fourier techniques and refined by a full matrix least-squares method (all hydrogen atoms were found by difference Fourier methods; $\mathrm{C}, \mathrm{O}$, and $\mathrm{N}$ atoms were refined with anisotropic temperature factors, $\mathrm{H}$ atoms with isotropic ones). The refinements are further characterized in Table I.

\section{Description of the structures}

Bond lengths, bond angles, and atom numbering are displayed in Fig. 1. Packing diagrams, presenting two different projections are contained in Figs. 2 and 3. Projections of individual stacks in a direction perpendicular to the plane of the BPN molecules are displayed in Fig. 4. Atomic coordi-

TABLE I. Lattice parameters and refinement.

\begin{tabular}{|c|c|c|c|c|}
\hline & \multirow{2}{*}{$\begin{array}{c}\text { BPN : TCNB } \\
\text { orange }\end{array}$} & \multicolumn{3}{|c|}{ BPN : PMDA } \\
\hline & & red & & \\
\hline Temperature (K) & 296 & 120 & 296 & 120 \\
\hline Crystal size (mm) & $0.5 \times 0.5 \times 1.0$ & $0.7 \times 0.5 \times 0.5$ & $1.0 \times 1.0 \times 0.5$ & $1.0 \times 1.0 \times 0.5$ \\
\hline Spacegroup & $P 2_{1} / a$ & $P 2_{1} / a$ & $P 2_{1} / a$ & $P 2_{1} / a$ \\
\hline$Z$ & 2 & 2 & 2 & 2 \\
\hline $2 \theta_{\max }(\mathrm{deg})$ & 55 & 65 & 70 & 70 \\
\hline $\sin \theta / \lambda_{\max }\left(\AA^{-1}\right)$ & 0.65 & 0.76 & 0.807 & 0.807 \\
\hline $\left.\begin{array}{l}\text { Number of reflections } \\
\text { for refining lattice }\end{array}\right\}$ & & & & \\
\hline parameters & 30 & 30 & 45 & 30 \\
\hline Angle range (deg) & $29.59<2 \theta<39.52$ & $30.09<2 \theta<44.78$ & $36.08<2 \theta<44.3$ & $35.10<2 \theta<44.54$ \\
\hline Scan range (deg) & 0.75 & 0.75 & 0.75 & 0.75 \\
\hline Scan rate $(\mathrm{deg} / \mathrm{min})$ & $2-24$ & $1-24$ & $2-24$ & $2-24$ \\
\hline$a(\AA)$ & $15.215(2)$ & $9.280(1)$ & $13.450(2)$ & $13.368(1)$ \\
\hline$b(\AA)$ & $7.260(1)$ & $11.869(2)$ & $5.917(1)$ & $5.809(1)$ \\
\hline$c(\AA)$ & $7.573(1)$ & $7.293(1)$ & $10.464(2)$ & $10.443(1)$ \\
\hline$\beta\left(^{\circ}\right)$ & $90.05(1)$ & $98.68(2)$ & $101.82(1)$ & $102.30(1)$ \\
\hline$V\left(\AA^{3}\right)$ & 836.5 & 794.0 & 815.1 & 792.3 \\
\hline$\rho_{\text {calc }}\left(\mathrm{g} / \mathrm{cm}^{3}\right)$ & 1.311 & 1.548 & 1.509 & 1.552 \\
\hline Unique reflections & 1889 & 2868 & 3568 & 3453 \\
\hline Contributing refl. & 1591 & 2456 & 2770 & 3203 \\
\hline Variables & 139 & 147 & 147 & 147 \\
\hline$R$ & 0.034 & 0.042 & 0.044 & 0.037 \\
\hline $\boldsymbol{R}_{w}$ & 0.047 & 0.053 & 0.060 & 0.056 \\
\hline$\sigma$ & 0.92 & 1.07 & 1.71 & 1.55 \\
\hline Extinc. cor. $\left(\times 10^{-3}\right)$ & 3.145 & none & 3.317 & 2.184 \\
\hline For the weighting sche & $+0.0125\left|F_{0}\right|+$ & $\left|F_{0}\right|^{2}+0.00001$ & & \\
\hline
\end{tabular}



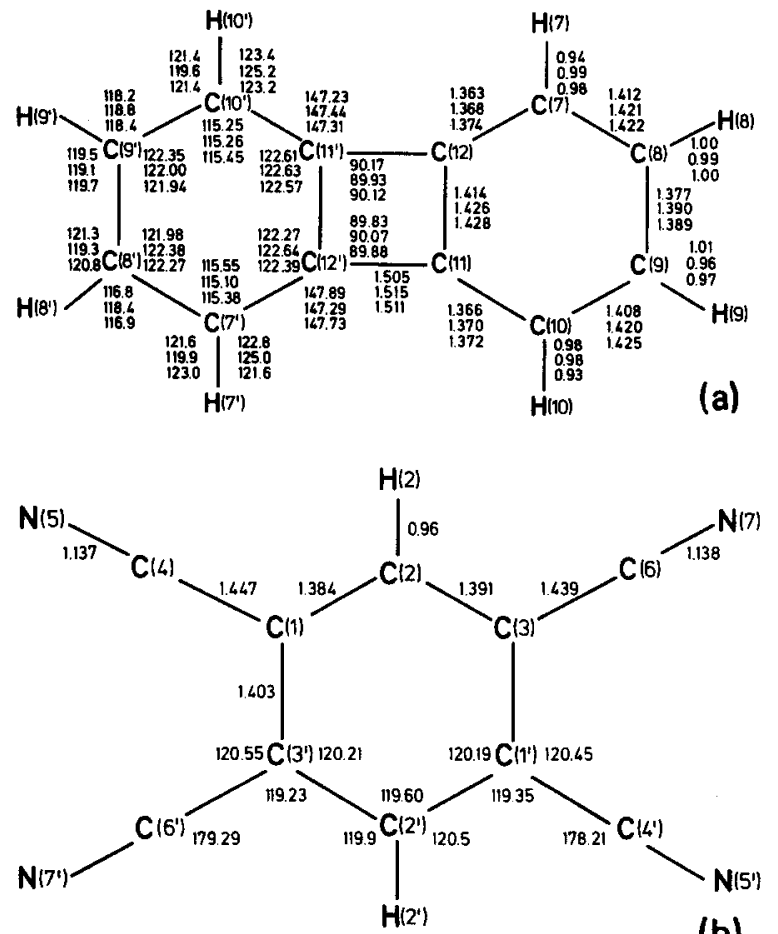

(b)

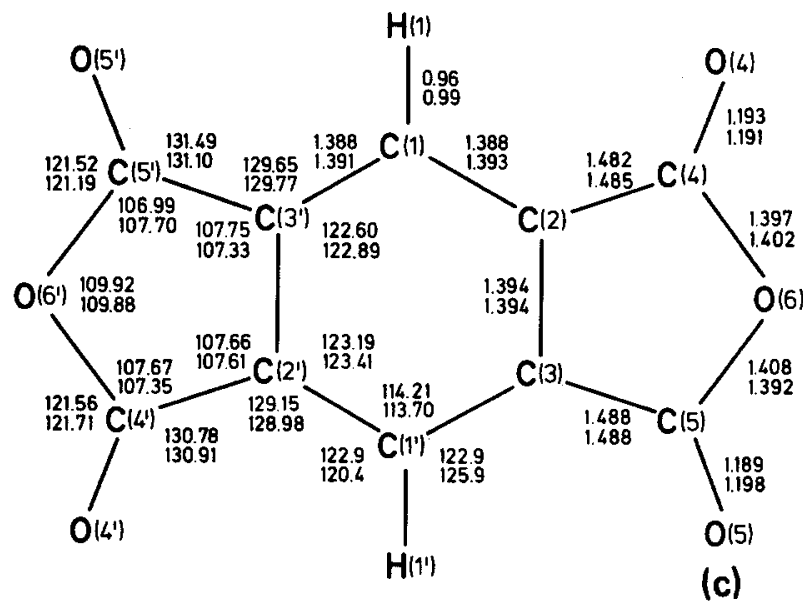

FIG. 1. (a) Atom numbering scheme, bond lengths, and bond angles of the BPN molecule. The upper value is for BPN:TCNB, the central for BPN:PMDA (red form), and the lowest for BPN:PMDA (black form). (b) Atom numbering scheme, bond lengths, and bond angles of the TCNB molecule in the complex BPN:TCNB. (c) Atom numbering scheme, bond lengths, and bond angles of the PMDA molecule. The upper values refer to the red modification, the lower numbers to the black.

nates and isotropic equivalent temperature factors are given in Table II. Equations for mean planes and long molecular axes are presented in Table III.

\section{BPN:TCNB}

The TCNB was arbitrarily placed in position $[0,0,0]$, the BPN in $[0,0,1 / 2]$. The structure is similar to, but deviates modestly from the usual scheme of mixed stacks of alternating donor and acceptor molecules (stacked with their planes parallel to each other), in that the molecular planes of BPN and TCNB are tilted with respect to each other at an angle of $\Phi=7.6^{\circ}$. The unit cell contains two stacks related by the symmetry operation $\frac{1}{2}+x, \frac{1}{2}-y, z$. Alternatively, the
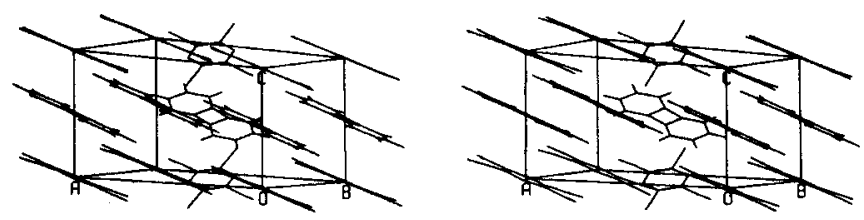

(a)
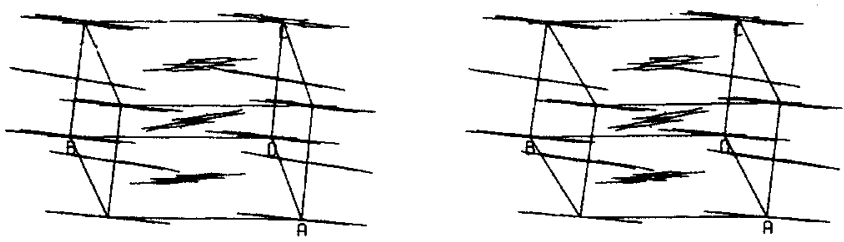

(b)
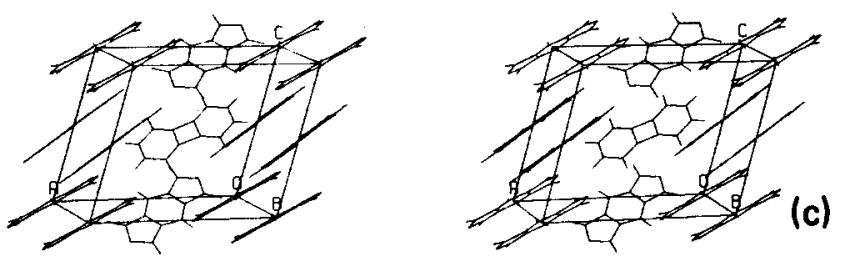

FIG. 2. Packing diagrams, stereoscopic projection viewed along a BPN plane for (a) BPN:TCNB, (b) BPN:PMDA (red form), (c) BPN:PMDA (black form).

structure can be viewed as consisting of alternating sheets, arranged parallel to (001), of BPN (at $z=1 / 2$ ) or TCNB (at $z=0$ ) molecules which are inclined with their molecular planes relative to the $(001)$ plane by $23.8^{\circ}$ and $24.5^{\circ}$, and relative to the molecular plane of their symmetry-related partner by $30.5^{\circ}$ and $41.6^{\circ}$, respectively. The packing within a layer is similar to that frequently found for molecular crystals such as naphthalene and anthracene ${ }^{13,14}$ (although the inclination angles within the layers are different for $N$ and $A$ ). The packing diagrams, Figs. 2(a) and 3(a), illustrate the sheet structure as well as the interstack interactions. The intrastack molecular overlap can be examined in Fig. 4(a).

\section{BPN:PMDA, red form}

The PMDA was placed in $[0,0,0]$, the BPN in $[0,0,1 / 2]$. Packing diagrams are shown in Figs. 2(b) and $3(\mathrm{~b})$, the projections of individual stacks in a direction perpendicular to the BPN molecular plane are displayed in Fig. 4(b) for comparison. The components form mixed stacks (with the $c$ axis as stack axis) in the usual manner. The normals of the donor and acceptor planes form an angle of $\Phi=4.1^{\circ}$, the long axes of BPN and PMDA are also nearly parallel, $\Omega=1.5^{\circ}$ (further details are given in Table III). The molecular plane normals are nearly parallel to the [001] stack axis.

\section{BPN:PMDA, black form}

The structure was determined for the same crystal at room temperature and at $120 \mathrm{~K}$ (see Table I). There are no unexpected differences, therefore only the low temperature 

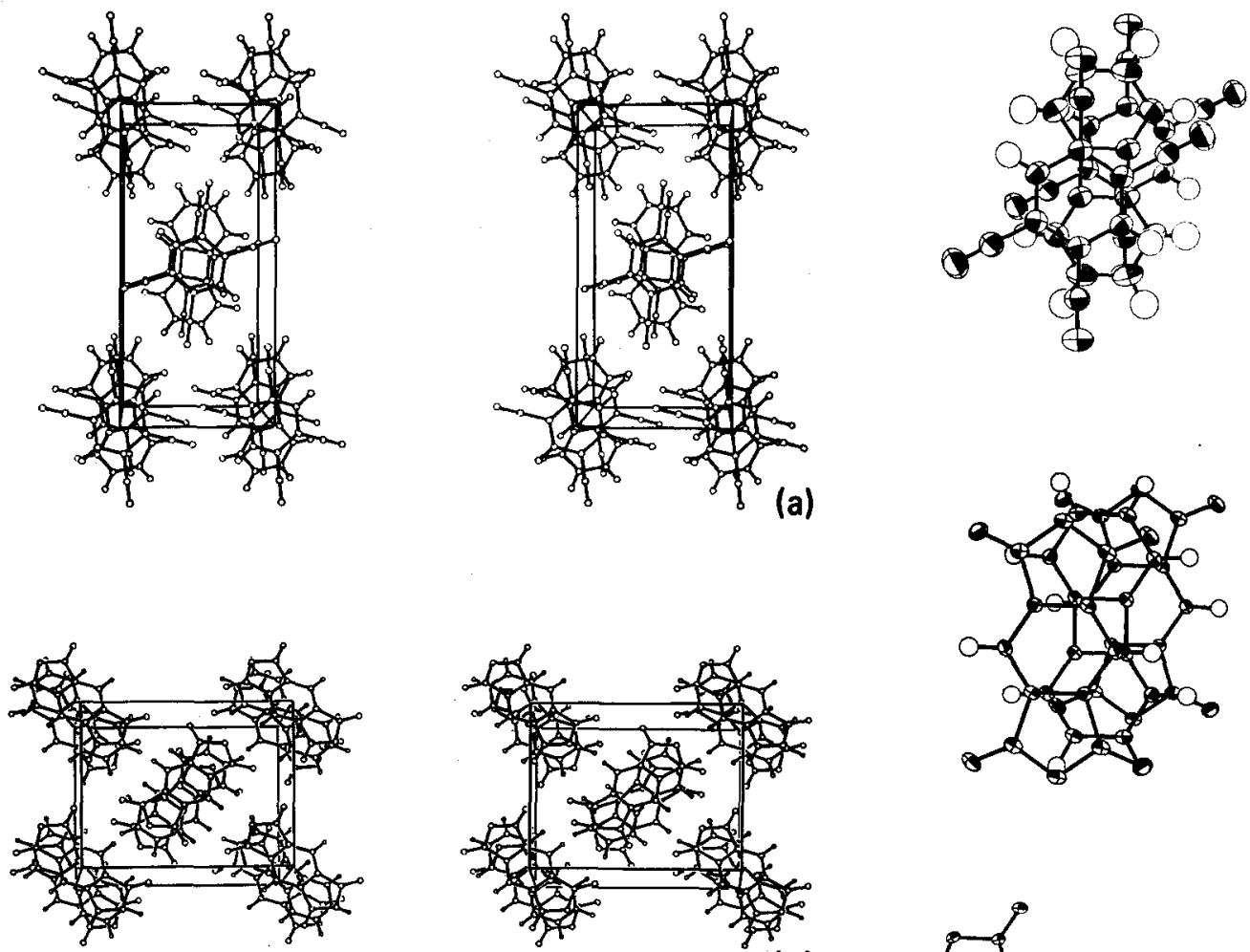

(b)
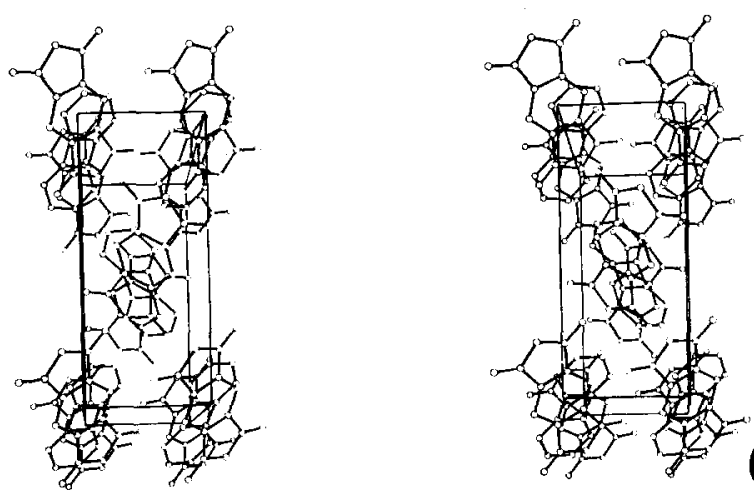

(c)

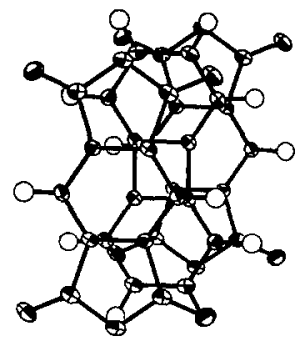
BPN:PMDA (black form).
FIG. 3. Packing diagrams, stereoscopic projection viewed onto a crystallographic $a b$ plane of (a) BPN:TCNB, (b) BPN:PMDA (red form), (c) BPN:PMDA (black form).

structure will be described. The angles between the mean molecular planes and the $c$ axis, which can be considered as the stack axis, are rather large: $46.3^{\circ}$ for PMDA and $52.2^{\circ}$ for BPN (details may be found in Table III). The donor and acceptor molecular planes are tilted with respect to each other at $\Phi=9.9^{\circ}$, which is an even larger deviation from the usual packing scheme than that described above for BPN:TCNB. The inclination angle of the molecular plane of the BPN donor molecules with respect to the $a b$-lattice plane $\left(42.0^{\circ}\right)$ and the corresponding angle of the acceptor molecules $\left(37.8^{\circ}\right)$ are also much larger than for BPN:TCNB $\left(23.8^{\circ}\right.$ and $24.5^{\circ}$, respectively). The distance between the center of a BPN molecule and the adjacent PMDA molecular plane is $3.301 \AA$; that between the center of a PMDA molecule and the adjacent BPN molecular plane is $3.197 \AA$.

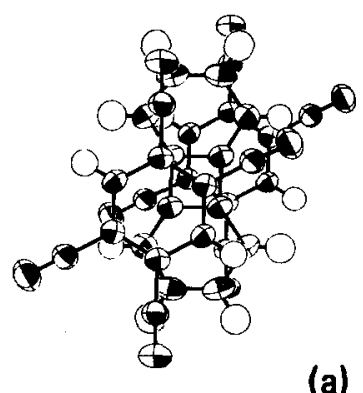

(a)

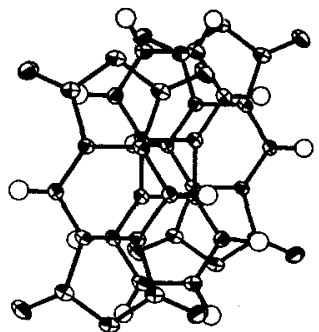

(b)
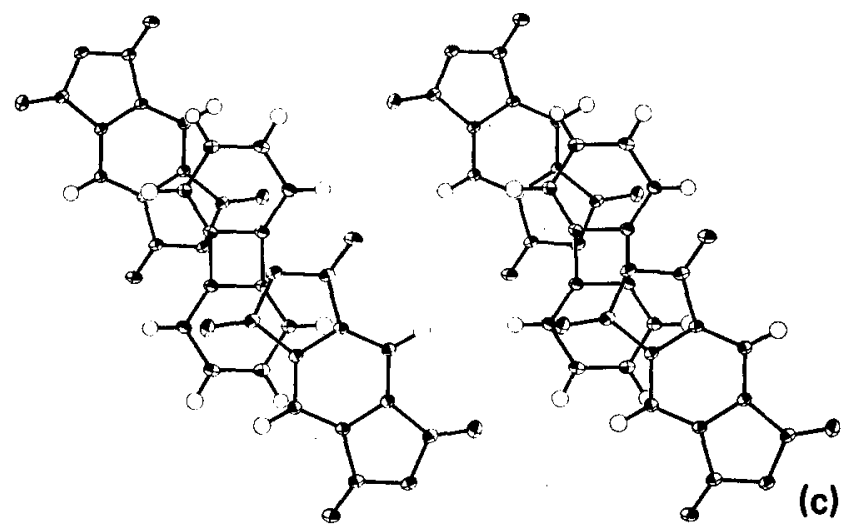

FIG. 4. Stacking of the complexes; stereoscopic projection viewed onto the BPN plane of (a) BPN:TCNB, (b) BPN:PMDA (red form), (c)

These distances are smaller than usual for charge transfer complexes; the small values are due to an inclination angle of $9.9^{\circ}$ in conjunction with the fact that the centers of the donor and acceptor molecules are shifted with respect to each other to an extent which is atypical for donor-acceptor complexes. Thus, PMDA overlaps one phenyl moiety of the BPN molecule with only part of one of its anhydride rings [Fig. 3(c) shows a view of this pseudostacking].

\section{CT absorption edges}

Room temperature absorption spectra, measured through plane parallel (natural) faces of small single crystals of the 1:1 complexes BPN:TCNB, BPN:PMDA (red form), and BPN:PMDA (black form) are plotted in Figs. 5 and 6. The curves displayed for each crystal refer to two principal extinction directions for normal incidence. The absolute extinction coefficients were determined from the relation $I=I_{0} e^{-\epsilon x}$, where $I_{0}$ is the incident, $I$ the transmitted 
TABLE II. List of atomic coordinates and equivalent isotropic temperature factors. [The temperature factor has the form of $\exp (-T)$, where $T=8 \pi^{2} \cdot U \cdot(\sin \theta / \lambda)^{2}$ for isotropic atoms. The estimated standard deviation of the last significant digit is given in parentheses. ]

\begin{tabular}{|c|c|c|c|c|}
\hline Atom & $X$ & $\boldsymbol{Y}$ & $Z$ & $U$ \\
\hline \multicolumn{5}{|c|}{ BPN : TCNB } \\
\hline $\mathrm{C}(1)$ & $-0.08734(9)$ & $0.0224(2)$ & $-0.0496(2)$ & 0.04200 \\
\hline$C(2)$ & $-0.06064(9)$ & $-0.1426(2)$ & $0.0245(2)$ & 0.04392 \\
\hline $\mathbf{H}(2)$ & $-0.102(1)$ & $-0.241(2)$ & $0.043(2)$ & 0.05530 \\
\hline$C(3)$ & $0.02666(9)$ & $-0.1655(2)$ & $0.0745(2)$ & 0.04245 \\
\hline C(4) & $-0.1786(1)$ & $0.0468(2)$ & $-0.0982(2)$ & 0.05150 \\
\hline$N(5)$ & $-0.25091(9)$ & $0.0630(2)$ & $-0.1328(2)$ & 0.07318 \\
\hline$C(6)$ & $0.0540(1)$ & $-0.3381(2)$ & $0.1500(2)$ & 0.05164 \\
\hline$N(7)$ & $0.0748(1)$ & $-0.4754(2)$ & $0.2096(2)$ & 0.07369 \\
\hline$C(7)$ & $-0.1022(1)$ & $0.2113(3)$ & $0.3730(2)$ & 0.05510 \\
\hline $\mathbf{H}(7)$ & $-0.093(1)$ & $0.336(2)$ & $0.346(2)$ & 0.05440 \\
\hline$C(8)$ & $-0.1838(1)$ & $0.1247(3)$ & $0.3411(2)$ & 0.06072 \\
\hline H(8) & $-0.231(1)$ & $0.202(3)$ & $0.287(2)$ & 0.07790 \\
\hline$C(9)$ & $-0.1981(1)$ & $-0.0577(3)$ & $0.3817(2)$ & 0.06181 \\
\hline $\mathbf{H}(9)$ & $-0.257(1)$ & $-0.115(3)$ & $0.356(2)$ & 0.07410 \\
\hline$C(10)$ & $-0.1326(1)$ & $-0.1700(3)$ & $0.4572(2)$ & 0.05646 \\
\hline$H(10)$ & $-0.144(1)$ & $-0.300(3)$ & $0.486(2)$ & 0.07330 \\
\hline $\mathrm{C}(11)$ & $-0.5392(9)$ & $-0.0850(2)$ & $0.4873(2)$ & 0.04597 \\
\hline$C(12)$ & $-0.03891(9)$ & $0.1024(2)$ & $0.4460(2)$ & 0.04570 \\
\hline \multicolumn{5}{|c|}{ BPN : PMDA (red form) } \\
\hline$C(1)$ & $-0.0948(1)$ & $-0.0921(1)$ & $-0.0721(2)$ & 0.01651 \\
\hline $\mathbf{H}(1)$ & $-0.158(2)$ & $-0.153(2)$ & $-0.117(3)$ & 0.02560 \\
\hline$C(2)$ & $0.0471(1)$ & $-0.1081(1)$ & $0.0158(2)$ & 0.01564 \\
\hline$C(3)$ & $0.1384(1)$ & $-0.0199(1)$ & $0.0855(2)$ & 0.01586 \\
\hline C(4) & $0.1300(1)$ & $-0.2145(1)$ & $0.0531(2)$ & 0.01888 \\
\hline $\mathbf{O ( 4 )}$ & $0.0952(1)$ & $-0.30978(8)$ & $0.0188(2)$ & 0.02561 \\
\hline$C(5)$ & $0.2806(1)$ & $-0.694(1)$ & $0.1690(2)$ & 0.01973 \\
\hline$O(5)$ & $0.3890(1)$ & $-0.0272(1)$ & $0.2468(2)$ & 0.02681 \\
\hline$O(6)$ & $0.2694(1)$ & $-0.18682(8)$ & $0.1426(1)$ & 0.02102 \\
\hline$C(7)$ & $0.0591(1)$ & $-0.1963(1)$ & $0.4890(2)$ & 0.01800 \\
\hline $\mathbf{H}(7)$ & $-0.001(2)$ & $-0.260(2)$ & $0.431(3)$ & 0.02840 \\
\hline$C(8)$ & $0.2033(1)$ & $-0.2163(1)$ & $0.5799(2)$ & 0.02042 \\
\hline $\mathbf{H}(8)$ & $0.242(2)$ & $-0.294(2)$ & $0.582(3)$ & 0.02760 \\
\hline$C(9)$ & $0.2924(1)$ & $-0.1304(1)$ & $0.6619(2)$ & 0.02045 \\
\hline $\mathbf{H}(9)$ & $0.391(2)$ & $-0.148(2)$ & $0.716(2)$ & 0.02400 \\
\hline$C(10)$ & $0.2450(1)$ & $-0.0166(1)$ & $0.6597(2)$ & 0.01862 \\
\hline$H(10)$ & $0.311(2)$ & $0.042(2)$ & $0.719(3)$ & 0.02480 \\
\hline$C(11)$ & $0.1059(1)$ & $0.0020(1)$ & $0.5715(2)$ & 0.01573 \\
\hline$C(12)$ & $0.0145(1)$ & $-0.0864(1)$ & $0.4880(2)$ & 0.01582 \\
\hline \multicolumn{5}{|c|}{ BPN : PMDA (black form) } \\
\hline$C(1)$ & $-0.05727(5)$ & $-0.2109(1)$ & $-0.04002(7)$ & 0.01402 \\
\hline $\mathbf{H}(1)$ & $-0.0940(8)$ & $-0.356(2)$ & $-0.071(1)$ & 0.02160 \\
\hline$C(2)$ & $0.03218(5)$ & $-0.1528(1)$ & $-0.08063(6)$ & 0.01371 \\
\hline$C(3)$ & $0.08705(5)$ & $0.0487(1)$ & $-0.04254(7)$ & 0.01336 \\
\hline$C(4)$ & $0.08638(5)$ & $-0.2815(1)$ & $-0.16839(7)$ & 0.01592 \\
\hline$O(4)$ & $0.06938(5)$ & $-0.4648(1)$ & $-0.21953(6)$ & 0.02199 \\
\hline$C(5)$ & $0.17750(5)$ & $0.0472(1)$ & $-0.10448(7)$ & 0.01586 \\
\hline$O(5)$ & $0.24818(4)$ & $0.1775(1)$ & $-0.09447(6)$ & 0.02155 \\
\hline$O(6)$ & $0.17105(4)$ & $-0.1489(1)$ & $-0.18233(5)$ & 0.01855 \\
\hline$C(7)$ & $-0.10010(5)$ & $0.1166(1)$ & $0.65460(7)$ & 0.01627 \\
\hline $\mathbf{H}(7)$ & $-0.0936(9)$ & $0.262(2)$ & $0.704(1)$ & 0.02440 \\
\hline$C(8)$ & $-0.17166(5)$ & $-0.0578(1)$ & $0.66875(7)$ & 0.01674 \\
\hline $\mathbf{H}(8)$ & $-0.2177(9)$ & $-0.025(2)$ & $0.731(1)$ & 0.01790 \\
\hline$C(9)$ & $-0.17845(5)$ & $-0.2644(1)$ & $0.60052(7)$ & 0.01754 \\
\hline$H(9)$ & $-0.229(1)$ & $-0.378(2)$ & $0.612(1)$ & 0.02260 \\
\hline$C(10)$ & $-0.11443(5)$ & $-0.3141(1)$ & $0.51104(7)$ & 0.01694 \\
\hline $\mathbf{H}(10)$ & $-0.1200(9)$ & $-0.453(2)$ & $0.465(1)$ & 0.01960 \\
\hline$C(11)$ & $-0.04590(5)$ & $-0.1448(1)$ & $0.49755(7)$ & 0.01506 \\
\hline$C(12)$ & $-0.03856(5)$ & $0.0669(1)$ & $0.56834(7)$ & 0.01488 \\
\hline
\end{tabular}

light intensity (corrected for reflection losses), and $x$ is the crystal thickness. The CT-absorption edges are broad and structureless, as is commonly encountered with weak donor-acceptor complexes. All samples exhibit dichroism, which is especially pronounced through the $(100)$ face of BPN:TCNB around $550 \mathrm{~nm}$. The spectra of the PMDA complexes displayed in Fig. 6, reflect in both polarizations essentially the same transition (albeit with different projections of the CT transition dipole moment on the two vibration directions, and hence with different intensity). In contrast, in the spectrum of BPN:TCNB, Fig. 5, there are two different absorption edges for the wave vector of the impinging light perpendicular to (100), one for the $E$ vector parallel to the stack axis $(E \|[001])$ and the other for $E$ perpendicular ( $E \|[010])$, a geometry for which crystal symmetry does not allow for a finite projection of an intrastack CT transition dipole moment on the [010] principal axis component of the optical tensor. The separation amounts to approximately $28 \mathrm{~nm}\left(850 \mathrm{~cm}^{-1} ; 0.11 \mathrm{eV}\right)$. For a rough characterization of the CT-threshold energy an extrapolation of the quasilinear portion of the CT-absorption edges down to $\epsilon=0$ may be used. With this definition the lower energy component lies at $588 \mathrm{~nm}(2.11 \mathrm{eV}), 565 \mathrm{~nm}(2.19 \mathrm{eV})$, and $690 \mathrm{~nm}(1.80 \mathrm{eV})$ for BPN:TCNB, BPN:PMDA (red form), and BPN:PMDA (black form), respectively.

\section{DISCUSSION}

\section{Comparison between the crystal structures of BPN:TCNB and of other similar TCNB complexes}

The complex N:TCNB, light yellow in color, has been described $^{1}$; the components are arranged in linear mixed stacks. It exhibits dynamic disorder of the naphthalene molecules $^{2,15}$ above a phase transition at $69^{16}$ or $63 \mathrm{~K},{ }^{17}$ characterized by libration of the long molecular axis by an angle of $\pm 18^{\circ}$. Anthracene forms a similar TCNB complex (red in color), which also exhibits dynamical disorder of the donor. ${ }^{6}$ A phase transition, which occurs on cooling below 206 $\mathrm{K}$, results in an ordered structure in which the angle between the long axes of symmetry-related anthracene molecules is temperature dependent. The red shift of the CT absorption edge in the series N:TCNB to BPN:TCNB to A:TCNB can be explained by the increasing size of the donor $\pi$-electron systems (for comparison Table IV gives the size and the molecular cell volume of the neat donor molecules and of their TCNB and PMDA complexes), and correlated with decreasing (gas phase) ionization energies in the series naphthalene, BPN, and anthracene. For a comparison of the vertical and adiabatic ionization energies in the gas phase and the ionization energies in the crystal see Table $V$. The absence of disorder in BPN:TCNB clearly indicates a more rigid fixation of the BPN donor, which can also be concluded from the fact that in the series of N, BPN, and A complexes BPN:TCNB has the highest density $\rho_{x}$, cf. Table IV.

The degree of overlap of donor and acceptor molecules is similar in all three complexes. The distance of the centers of gravity of the donor molecules and the planes of adjacent acceptor molecules are also very similar: N:TCNB (3.43 $\AA$, A:TCNB (3.42 Å), and BPN:TCNB (3.45 $\AA$ ).

\section{Comparison of the crystal structures of BPN:PMDA with those of other similar PMDA complexes}

The crystal structures of BPN:PMDA can be compared to the two forms of N:PMDA. ${ }^{3,4}$ The red form of 
TABLE III. Least-squares planes and long molecular axes. The $i, j$, and $k$ axes of the orthonormal coordinate system (in $\AA$ space) are in directions: $i$ parallel $a, j$ parallel $b$, and $k$ parallel $c^{*}$. The angles are defined as follows: $\Phi=$ angle between the normals to the donor and acceptor molecular planes, $\Psi=$ tilt angle of donor and acceptor normals with respect to the [001] stacking direction, $\Omega=$ angle between the long axes of the donor and acceptor molecules.

(1) BPN : TCNB:

Equation of the TCNB plane:

$-0.214 i+0.355 j+0.910 k=0.000$

Equation of the BPN plane:

$-0.308 i+0.262 j+0.914 k=3.463$

Distance between center of TCNB

and next BPN plane: $3.461 \AA$

Distance between center of BPN

and next TCNB plane: $3.454 \AA$

$$
\begin{aligned}
& \Phi=7.6^{\circ} \\
& \Psi_{D}=23.8^{\circ} \\
& \Psi_{A}=24.5^{\circ}
\end{aligned}
$$

Equation of the long axis of

TCNB, defined by atoms $\mathrm{C}(1)$,

$C(4)$, and $N(5)$ :

$$
\begin{array}{lr}
i= & 0.959 t \\
j= & -0.117 t \\
k= & 0.257 t
\end{array}
$$

Equation of the long axis of

BPN, defined by center between atoms $\mathrm{C}(8), \mathrm{C}(9)$, and between atoms $C(11)$ and $C(12)$ :

$$
\begin{aligned}
& i=-0.003+0.938 t \\
& j=0.079 t \\
& k=3.786+0.339 t \\
& \mathbf{\Omega}=5.3^{\circ}
\end{aligned}
$$

(2) BPN : PMDA (red form):

Equation of the PMDA plane:

$-0.467 i-0.080 j+0.880 k=0.000$

Equation of the BPN plane:

$-0.486 i-0.147 j+0.861 k=3.373$

Distance between center of PMDA

and next BPN plane: $3.373 \AA$

Distance between center of BPN

and next PMDA plane: $3.431 \AA$

$$
\begin{aligned}
& \Phi=4.1^{\circ} \\
& \Psi_{D}=22.3^{\circ} \\
& \Psi_{A}=19.8^{\circ}
\end{aligned}
$$

Equation of the long axis of

PMDA, defined by atom $O(6)$ and

center between atoms $C(2)$ and C(3):

$$
\begin{array}{lr}
i= & -0.692 t \\
j= & 0.655 t \\
k= & -0.305 t
\end{array}
$$

Equation of the long axis of

BPN, defined by centers between

atoms $\mathrm{C}(8), \mathrm{C}(9)$ and between

$\mathrm{C}(11)$ and $\mathrm{C}(12)$ :

$$
\begin{array}{lr}
i= & -0.551-0.696 t \\
j= & 0.661 t \\
k= & 3.605-0.280 t \\
\Omega= & 1.5^{\circ}
\end{array}
$$

\section{(3) BPN : PMDA (black form):}

Equation of the PMDA plane:

$0.382 i-0.479 k+0.790 k=0.000$

Equation of the BPN plane:

$0.531 i-0.408 j+0.743 k=3.197$

Distance between center of PMDA

and next BPN plane: $3.197 \AA$

Distance between center of BPN and next PMDA plane: $3.301 \AA$

$$
\begin{aligned}
& \Phi=9.9^{\circ} \\
& \Psi_{D}=52.2^{\circ} \\
& \Psi_{A}=46.3^{\circ}
\end{aligned}
$$

Equation of the long axis of PMDA, defined by atom $O(6)$ and center between atoms $C(2)$ and $\mathrm{C}(3)$ :

$$
\begin{array}{cc}
i= & 0.796 t \\
j= & -0.256 t \\
k= & -0.548 t
\end{array}
$$

Equation of the long axis of BPN, defined by center between atoms $\mathrm{C}(8), \mathrm{C}(9)$ and between atoms $C(11)$ and $C(12)$ :

$$
\begin{array}{ll}
i= & -1.112+0.846 t \\
j= & 0.300 t \\
k= & 5.102-0.441 t \\
\Omega=33.0^{\circ} &
\end{array}
$$

BPN:PMDA is similar to the yellow, ordered form of $\mathrm{N}: \mathrm{PMDA}^{3}$ insofar as there is the same stacking architecture with similar angles, a similar eclipsing pattern and no disorder (cf. Ref. 4). The red shift of the color is a result of the larger size of the BPN- $\pi$-electron system compared to $N$.

The structure of black BPN:PMDA is similar to that of the orange low temperature $\left(T<188 \mathrm{~K}^{4}\right)$ ordered form of $\mathrm{N}: \mathrm{PMDA},{ }^{4}$ (the high temperature form of N:PMDA is disordered). However, the angles of tilt to the "stack axis"

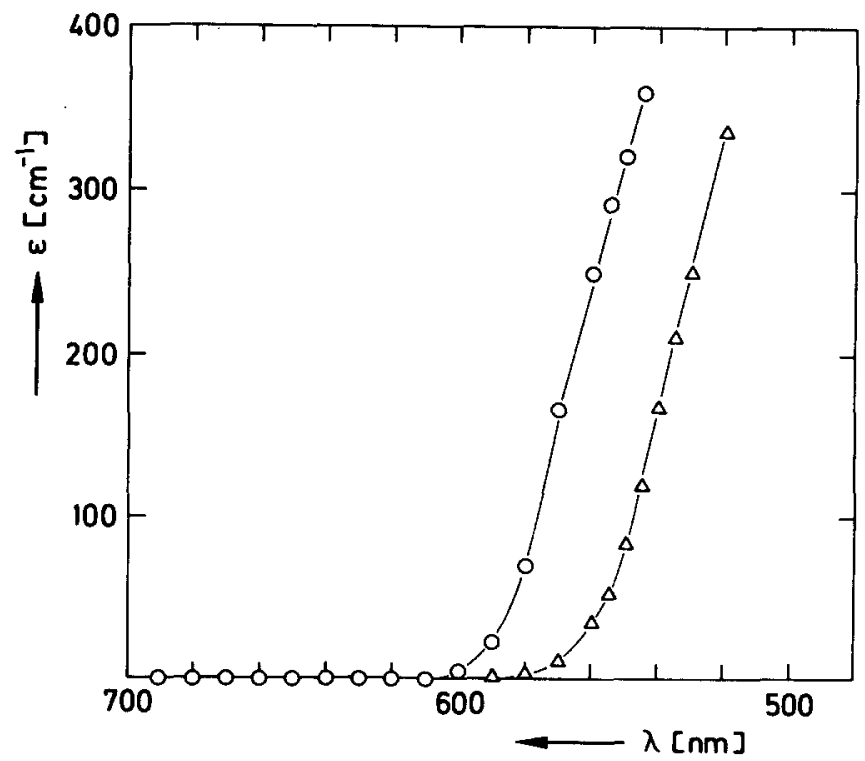

FIG. 5. Absorption spectra of BPN:TCNB at room temperature for the two principal extinction directions, $E$ parallel to the stack axis, $E \| c$ (circles), and $E \| b$ (triangles), at normal incidence on (100). The crystal used for the measurement had the dimensions $0.6 \times 0.5 \times 0.145 \mathrm{~mm}^{3}$.
[001] are greater in BPN:PMDA (52.2 $2^{\circ}$ and $46.3^{\circ}$ for BPN and PMDA, respectively) than in N:PMDA $\left(37^{\circ}\right) .{ }^{34}$ The black color will be tentatively explained in the following paragraph.

\section{Energy of the charge transfer transitions}

The charge transfer transition threshold energy $E_{C T}^{\text {th }}$ in weak charge transfer crystals (i.e., with more or less neutral ground state) is given by the minimum energy which is need-

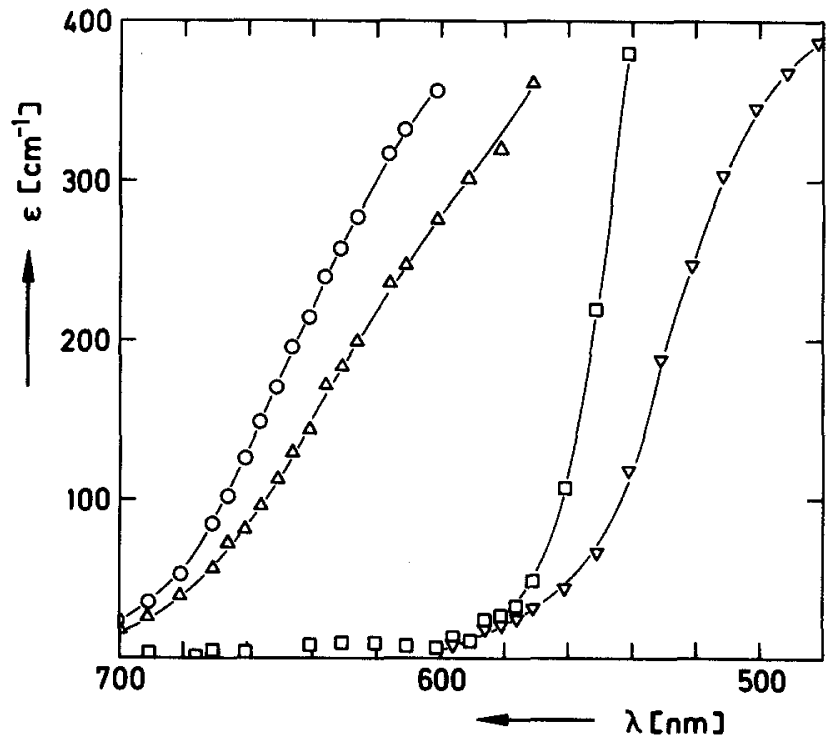

FIG. 6. Absorption spectra of BPN:PMDA for the two principal extinction directions of arbitrarily oriented crystals at room temperature. The symbols $\square$ and $\nabla$ refer to the red form; $\Delta$ and $O$ to the black modification. 
TABLE IV. Trends of the unit cell volumes per formula unit, $V_{0} / 2$, and the $x$-ray densities, $\rho_{x}$, in the series of TCNB and PMDA complexes of naphthalene, $\mathrm{C}_{10} \mathrm{H}_{8}$, biphenylene, $\mathrm{C}_{12} \mathrm{H}_{8}$, and anthracene, $\mathrm{C}_{14} \mathrm{H}_{10}$, at the temperatures specified. The donor size is characterized by its long axes $\mathrm{l}_{D}$ (Ref. 33 ), its neat crystal cell volume per one molecule, $V_{\alpha} / 2$, and its $x$-ray density, $\rho_{x}$.

\begin{tabular}{|c|c|c|c|c|c|c|c|c|c|c|}
\hline & \multicolumn{3}{|c|}{$\begin{array}{l}\text { 1:1 complex } \\
\text { with TCNB }\end{array}$} & \multicolumn{3}{|c|}{$\begin{array}{l}\text { 1:1 complex } \\
\text { with PMDA }\end{array}$} & \multirow{2}{*}{$\begin{array}{l}\text { Donor } \\
\text { long axis } \\
1_{D} \text { (A) }\end{array}$} & \multicolumn{3}{|c|}{ Neat donor crystal } \\
\hline & $T(\mathbf{K})$ & $V_{0} / 2\left(\AA^{3}\right)$ & $\rho_{x}\left(\mathrm{~g} / \mathrm{cm}^{3}\right)$ & $T(\mathbf{K})$ & $V_{0} / 2\left(\AA^{3}\right)$ & $\rho_{x}\left(\mathrm{~g} / \mathrm{cm}^{3}\right)$ & & $T(\mathbf{K})$ & $V_{0} / 2\left(\AA^{3}\right)$ & $\rho_{x}\left(\mathrm{~g} / \mathrm{cm}^{3}\right)$ \\
\hline $\mathbf{N}$ & 296 & $390.1^{\mathrm{a}}$ & 1.304 & $\begin{array}{l}293 \\
153\end{array}$ & $\begin{array}{l}393.5^{b} \\
383.5^{c}\end{array}$ & $\begin{array}{l}1.460 \\
1.498\end{array}$ & 8.55 & 109 & $172.5^{\mathrm{i}}$ & 1.234 \\
\hline BPN & 296 & $418.3^{d}$ & 1.311 & $\begin{array}{l}120 \\
120\end{array}$ & $\begin{array}{l}397.0^{e} \\
396.2^{f}\end{array}$ & $\begin{array}{l}1.548 \\
1.552\end{array}$ & 10.03 & 296 & $203.8^{j}$ & 1.240 \\
\hline $\mathbf{A}$ & 297 & $452.6^{8}$ & 1.307 & 153 & $429.6^{h}$ & 1.532 & 11.00 & 95 & $229.2^{k}$ & 1.286 \\
\hline
\end{tabular}

"Reference 2.

'Yellow complex (Ref. 3).

'Orange complex (Ref. 4).

dThis work.

'This work, red form.

'This work, black form.
${ }^{8}$ Reference 6.
${ }^{b}$ Reference 21.
${ }^{\mathrm{i}}$ Reference 18.
${ }^{\mathrm{j}}$ Reference 19.
${ }^{\star}$ Reference 20.

ed to remove an electron from the donor molecule (in the complex crystal) to infinity (the so-called adiabatic solid state ionization threshold, $I_{D}^{a}$ ), minus the energy which is gained from an attachment of the electron to a noninteracting distant acceptor molecule (again in the complex crystal), (the adiabatic solid state electron affinity, $A_{A}^{a}$ ) minus the Coulombic binding energy, $C$ which the resulting $\mathrm{D}^{+} \ldots \mathrm{A}^{-}$pair gains when the separation is reduced to the finite distance of the considered D:A pair, minus a rearrangement energy, $R$ (due to vibronic molecular and phononic lattice relaxation ), and a change, $\Delta$, of the polarization, van der Waals and exchange interactions, (with respect to the neutral lattice) on pair formation

$$
E_{\mathrm{CT}}^{\text {th }}=I_{D}^{a}-A_{A}^{a}-C-R-\Delta .
$$

In a first order approximation we take $R=0$ and $\Delta=0$, and assume $I_{D}^{a}$ to equal the adiabatic ionization threshold of the donor in the neat donor crystal, and $A_{A}^{a}$ to equal to the adiabatic electron affinity of the acceptor in the neat acceptor crystal. With these simplifications it is possible to predict the energy of the threshold of the lowest charge transfer transition which we are interested in.

The assumption $R=0$ is reasonable because the charge

TABLE V. Comparison of ionization energies of naphthalene (N), biphenylene (BPN), and anthracene (A): ( $v=$ vertical, $a=$ adiabatic).

\begin{tabular}{ccccc}
\hline \hline & $I_{D}^{\mathrm{v}}$ (gas) (eV) & $I_{D}^{\mathrm{a}}$ (gas) (eV) & $I_{D}^{\mathrm{a}}$ (crystal) (eV) $P_{i}(\mathrm{eV})$ \\
\hline $\mathrm{N}$ & $8.15^{\mathrm{a}}$ & $8.12^{\mathrm{a}, \mathrm{b}}$ & $6.4^{\mathrm{b}}$ & 1.72 \\
$\mathrm{BPN}$ & $7.61^{\mathrm{c}}$ & $\ldots$ & $5.92^{\mathrm{d}}$ & $1.65^{\mathrm{e}}$ \\
$\mathrm{A}$ & $7.41^{\mathrm{a}, \mathrm{f}}$ & $7.36^{\mathrm{a}}$ & $5.75(10)^{\mathrm{g}}$ & 1.61 \\
\hline
\end{tabular}

Reference 22.

'Reference 23.

' References 24 and 25.

d Calculated from $I_{D}-P_{i}$.

- Interpolated between the $\mathrm{N}$ and $\mathrm{A}$ values.

${ }^{\text {'Reference } 26 .}$

- Reference 27. transfer threshold energy (as defined above) should reflect the purely electronic transition before lattice relaxation takes place, however strong the electron phonon coupling may be [i.e., before the Coulombic attraction between $\mathrm{D}^{+}$ and $\mathrm{A}^{-}$leads to a change in vibrational frequencies and to a new spatial equilibrium position of the ionized donoracceptor molecular pair (or $\mathrm{D}^{+1 / 2} \mathrm{~A}^{-} \mathrm{D}^{+1 / 2}$ or $A^{-1 / 2} D^{+} A^{-1 / 2}$ trimer, cf. Refs. 2 and 28 ) with locally changed phonon frequencies].

The Coulomb energy $C$ is a function of the spatial part of the radical cation and radical anion wave functions (i.e., of the charge distribution on the $\mathrm{D}^{+}$and $\mathrm{A}^{-}$molecule), of the interaction distance, of screening by the other polarizable molecules (being in their ground state), of delocalization, and of the degree of charge transfer, which is not necessarily a full electron. With these basic considerations in mind, let us now try to arrive at a semiquantitative interpretation.

Since for BPN only the gas phase vertical (unrelaxed) ionization energy is available $\left(I_{\mathrm{BPN}}^{v}=7.61 \mathrm{eV}^{24,25}\right)$ we have to derive the solid state value by comparison with the next smaller and larger linear condensed polycyclic aromatic hydrocarbons, $\mathbf{N}$ and $\mathbf{A}$, cf. Table $\mathbf{V}$. It is seen that the differences between their respective vertical and adiabatic gas phase ionization energies are small (at least with respect to the experimental reproducability ), a fact which allows a reasonable estimate of $I_{\mathrm{BPN}}^{a}$ (gas) to be made $(\sim 7.57 \mathrm{eV})$. An approximate value of the BPN solid state ionization threshold can be obtained by assuming a polarization shift $P_{i}=I_{\mathrm{BPN}}^{a}$ (gas) $-I_{\mathrm{BPN}}^{a}$ (crystal) of $1.65 \mathrm{eV}$ (obtained by interpolation between the naphthalene and anthracene values) (cf. Ref. 23):

$$
I_{\mathrm{BPN}}^{a}(\text { crystal })=I_{\mathrm{BPN}}^{a}(\text { gas })-P_{i}=5.92 \mathrm{eV} .
$$

It has recently been demonstrated by photoelectron spectroscopy of several weak donor-acceptor complexes with the acceptor PMDA that in fact the ionization threshold of a donor such as anthracene is by $0.2-0.3 \mathrm{eV}$ higher in the complex than in the neat donor crystal $^{27}$ (cf. Table V). Taking this shift into account we estimate $I_{\mathrm{BPN}}^{a}$ in the BPN:PMDA complex to amount to $\sim 6.17 \mathrm{eV}$. 
The solid state electron affinity of PMDA has been obtained only indirectly. But the sum $-A_{A}^{a}-C-R-\Delta$ of Eq. (1) has been found to be $-3.88 \pm 0.15 \mathrm{eV}$ for the series of the 1:1 PMDA complexes of phenothiazine, anthracene, carbazole, and acridine ${ }^{27}$; in analogy, a similar value may be expected for BPN:PMDA. Therefore $E_{\mathrm{CT}}$ (BPN:PMDA) is predicted as $6.17-3.88=2.29 \pm 0.15 \mathrm{eV}$. This value is in fact in good agreement with the experimental result of the red modification of BPN:PMDA (which forms regular mixed stacks), $E_{\mathrm{CT}}=2.19 \mathrm{eV}$, if one takes into account the energetic uncertainty which arises from the crude definition of the CT energy from the absorption spectrum (as made above), together with the fact that at room temperature the CT absorption edges are not very sharply definable anyway. It is difficult to further deconvolute the sum $-A_{A}^{a}$ $-C-R-\Delta$. However, $\delta=(-C-R-\Delta)$ should be reflected by the thermal activation energy needed to separate an (optically) created CT pair into independently mobile "free" charge carriers (to "infinite" distance), and therefore can be obtained approximately from the activation energy of the photoconduction quantum yield. Available values are 0.14(3) eV for A:PMDA, ${ }^{29} 0.5$ (1) eV for phenanthrene:PMDA. ${ }^{28(c)}$ More indirectly, the difference between the separation of the valence band and the conduction band (band gap energy, obtainable from the dark conductivity activation energy), and the CT transition energy can be used, leading to $\delta=0.26(6) \mathrm{eV}$ for phenothiazine: PMDA ${ }^{8,27}$ With an average of $\delta=0.3 \mathrm{eV}$, the electron affinity of PMDA in PMDA complexes can be estimated to amount to $3.6(3) \mathrm{eV}$.

We now consider the surprisingly strong bathochromic shift $\Delta E_{\mathrm{CT}}$ of the CT edge at the phase transformation from the usual mixed stack red modification to the layer-packed, black BPN:PMDA modification, $\Delta E_{\mathrm{CT}}=2.19-1.80$ $=0.39 \mathrm{eV}$, which demonstrates a stronger CT interaction in the black form. The origin of this shift must result from the influence of structure on one or more of the terms in Eq. (1).

What structural differences may be relevant? In terms of the conventional understanding of charge transfer interaction, the structural properties of the black modification appear less favorable for significant charge transfer. The molecular planes of the donor and acceptor molecules within a stack are tilted by $9.9^{\circ}$ to each other. Because of the large inclination of the stack direction, [001], to the molecular plane normals ( $52.2^{\circ}$ for BPN, $46.3^{\circ}$ for PMDA), the centers of the donor and acceptor molecules are unusually far apart, $5.23 \AA$. This results in only modest molecular overlap of those regions of the molecules carrying the $\pi$-electron density: $C(7)$ and $C(8)$ of BPN with $C(2)$ of PMDA (3.337 and $3.397 \AA$, symmetry operator $x, y, z-1), C(10)$ of BPN with $O(4)$ of PMDA (3.432 $\AA$, symmetry operator $x, y, z-1)$, and $C(12)$ of BPN and $O(6)$ of PMDA (3.619 $\AA$, symmetry operator $x, y, z-1)$. The interactions are confined to the ends of the donor and acceptor molecules and are repeated by the crystallographic inversion centers. In contrast, in the red form a considerable fraction of the atoms carrying $\pi$ electrons are in close intrastack intermolecular proximity [C(7) of BPN with C(4) of PMDA (3.349 $\AA$ ); $\mathrm{C}(8)$ with $\mathrm{O}(6)$ (3.356 $\AA$ ); $\mathrm{C}(11)$ with $\mathrm{C}(3)$ (3.612 $\AA$ );
$\mathrm{C}(12)$ with $\mathrm{C}(2)(3.511 \AA) ; \mathrm{C}\left(10^{\prime}\right)$ with $\mathrm{C}\left(3^{\prime}\right)$ (3.398 $\AA$, symmetry operator $x, y, z-1)$, and $\mathrm{C}\left(12^{\prime}\right)$ with $\mathrm{C}\left(1^{\prime}\right)$ (3.508 $\AA$, symmetry operator $x, y, z-1$ ) in $c$ direction]. Furthermore, the molecular planes are parallel to within $4.1^{\circ}$ and the center of the BPN molecular is only $3.43 \AA$ from the center of the PMDA molecule.

In the black modification, however, the arrangement of an alternating sequence of inclined donor molecules in donor layers and acceptor molecules in acceptor layers [cf. Fig. 2(c)] provides a possibility for considerable donor-donor and acceptor-acceptor interstack (transverse) $\pi$ interactions allowing for additional two-dimensional lateral delocalization of the interacting electron and hole, and hence a bathochromic shift of the CT transition.

Further, considering a donor BPN molecule with its long axis designated $L$, its medium axis (in plane) $M$, and its short axis (normal to the molecular plane), $N$, the two adjacent (intrastack) PMDA acceptor molecules are shifted (in opposite directions) from the BPN molecule approximately along the axis $M$ in the red modification, whereas in the black form they are shifted essentially along the axis $L$ (of the acceptor), cf. Figs. 4(b) and 4(c).

Depending on the symmetry of the donor HOMO and acceptor LUMO electronic wave functions these two situations may lead to completely different interferences and hence interactions of the $\pi$-electron wave functions. In addition, interactions with the BPN $\pi$-electron system occur in the more polarizable direction $(L)$ in the black complex as compared to the less polarizable $M$ direction in the red one.

Recently it has been argued ${ }^{28(c)}$ that orbital symmetry should play an important role in CT interaction. We believe that both orbital symmetry and polarizability are fundamental properties that need more experimental work to elucidate their relative importance.

Finishing our considerations of the two BPN:PMDA complexes we should not leave one important aspect unmentioned: Both structures have very nearly the same unit cell volume, 794.0 and 792.3 $\AA^{3}$ (at $\sim 120 \mathrm{~K}$ ) for the (undercooled) red and (stable) black form, respectively, an observation, which indicates that simple packing density considerations are not adequate.

A brief discussion of the BPN:TCNB CT-absorption edge follows. It lies at a slightly lower energy $(2.11 \mathrm{eV})$ than that of red BPN:PMDA (2.19 eV), although TCNB is believed to be a slightly weaker acceptor than PMDA $A_{\text {TCNB. }}$ (gas) $=2.00 \mathrm{eV},{ }^{30} A_{\mathrm{PMDA}}$ (gas) $=2.04 \mathrm{eV}^{30}$ ], an assumption which is strongly supported by a comparison of the 4.2 $\mathrm{K}$ CT-absorption onset of the complexes A:PMDA (2.27 $\mathrm{eV}^{31}$ ) and A:TCNB $\left(2.41 \mathrm{eV}^{32}\right)$. Therefore, in contradiction to the result of simple theoretical considerations

$$
\begin{aligned}
\left(E_{\mathrm{CT}}\right. & \propto\left[I_{D} \text { (crystal) }-A_{A} \text { (crystal) }\right] \\
& \left.\sim\left[I_{D} \text { (gas) }-A_{A} \text { (gas) }\right]\right),
\end{aligned}
$$

there is not a hypsochromic, but a small bathochromic shift of the CT edge in the real BPN:TCNB complex with respect to that in BPN:PMDA. This might be understood on the basis of the structure of this complex which also exhibits the type of packing with alternate layers of inclined donor and acceptor molecules, for which an increase of dimensionality 
of the CT interaction was inferred for the black BPN:PMDA complex above. Since the intrastack donor-acceptor molecular overlap in BPN:TCNB is normal, it appears reasonable to assume an increased dimensionality of the $\mathrm{CT}$ interaction as cause for the observed bathochromic shift. In view of these considerations we assign the higher energy absorption edge for the electric light vector $E$ perpendicular to the stack axis [and perpendicular to the (010) symmetry plane], cf. Fig. 5, to an interstack charge transfer transition (transfering the electron to a more distant acceptor than the intrastack next neighbor).

In conclusion, we have prepared and characterized three new donor-acceptor complexes between the donor biphenylene and the acceptors TCNB and PMDA and have found packing schemes with two-dimensional interactions in donor layers and in acceptor layers which imply the interesting possibility of three-dimensional delocalization of the CT state that may give rise to three-dimensionally mobile CT excitons. These unusual structural features should also be reflected in the charge carrier mobility tensors and in the mechanism of charge carrier (pair) generation. These very interesting aspects deserve further investigation.

Note added in proof: See AIP Document No. PAPS JCPSA-84-5162-58, for 58 pages of structure factor tables for BP-TCNB.

\section{ACKNOWLEDGMENTS}

We wish to thank Dr. R. Radomski, Technical University of Wroclaw, Poland, who did part of the DSC investigations during a stay at the Physics Institute of the University of Stuttgart. TCNB was synthesized and purified by W. Tuffentsammer; PMDA and BPN were zone refined by $M$. Gerdon and Chr. M. Herb. This work was supported in large part by the Stiftung Volkswagenwerk and in part by the Verband der Chemischen Industrie e.V.

'S. Kumakura, F. Iwasaki, and Y. Saito, Bull. Chem. Soc. Jpn. 40, 1826 (1967).

${ }^{2}$ A. Grupp, Doktorarbeit, Universität Stuttgart, 1982.

${ }^{3} \mathrm{M}$. Le Bars-Combe, B. Chion, and J. Lajzérowicz-Bonneteau, Acta Crystallogr. Sect. B 35, 913 (1979).

${ }^{4} \mathrm{M}$. Le Bars-Combe and J. Lajzérowicz-Bonneteau, Acta Crystallogr. Sect. B 37, 1707 ( 1981 ).
${ }^{5}$ H. Tsuchiya, F. Marumo, and Y. Saito, Acta Crystallogr. Sect. B 28, 1935 (1982).

бJ. J. Stezowski, J. Chem. Phys. 73, 538 (1980).

${ }^{7} \mathrm{M}$. Gerdon and N. Karl (unpublished).

${ }^{8}$ R. Antonj, N. Karl, B. E. Robertson, and J. J. Stezowski, J. Chem. Phys. 72, 1244 (1980).

${ }^{9}$ W. Tuffentsammer (unpublished).

${ }^{10} \mathrm{~N}$. Karl, in Crystals, Growth Properties, and Applications, edited by H. C. Freyhardt (Springer, Berlin, 1980), Vol. 4, pp. 1-100.

${ }^{11}$ J. M. Stewart, P. A. Machin, C. W. Dickinson, H. L. Ammon, H. Flack, and H. Heck, X-Ray System, Version of 1976 Technical Report TR-446, Computer Science Center, University of Maryland, College Park, Maryland.

${ }^{12}$ P. Main, L. Lessinger, M. M. Woolfson, G. Germain, and J.-P. Declerq, MULTAN 80, A Program for the Automatic Solution of Crystal Structures from X-Ray Diffraction Data, University of York, England, 1980.

${ }^{13}$ S. C. Abrahams, J. M. Robertson, and J. G. White, Acta Crystallogr. 2, 233 (1949).

${ }^{14}$ V. C. Sinclair, J. M. Robertson, and A. M. Mathieson, Acta Crystallogr. 3, 251 (1950).

${ }^{15}$ R. M. Macfarlane and S. Ushioda, J. Chem. Phys. 67, 3214 (1977).

${ }^{16}$ H. J. Bernstein, N. S. Dalal, W. F. Murphy, A. H. Reddoch, S. Sunder, and D. F. Williams, Chem. Phys. Lett. 57, 159 (1978).

${ }^{17}$ E. Erdle and H. Möhwald, Chem. Phys. 36, 283 (1979).

${ }^{18}$ C. P. Brock and J. D. Dunitz, Acta Crystallogr. Sect. B 38, 2218 (1982).

${ }^{19}$ J. K. Fawcett and J. Trotter, Acta Crystallogr. 20, 87 (1966).

${ }^{20}$ R. Mason, Acta Crystallogr. 17, 547 (1964).

${ }^{21}$ B. E. Robertson and J. J. Stezowski, Acta Crystallogr. Sect. B 34, 3005 (1978).

${ }^{22}$ P. A. Clark, F. Brogli, and E. Heilbronner, Helv. Chim. Acta 55, 1415 (1972).

${ }^{23}$ N. Sato, K. Seki, and H. Inokuchi, J. Chem. Soc. Faraday Trans 277 , 1621 (1981).

${ }^{24}$ R. Boschi, E. Clar, and W. Schmidt, J. Chem. Phys. 60, 4406 (1974).

${ }^{25}$ J. P. Maier and D. W. Turner, Faraday Discuss. Chem. Soc. 54, 149 (1972).

${ }^{26}$ W. Schmidt, J. Chem. Phys. 66, 828 (1977).

${ }^{27}$ N. Karl, N. Sato, K. Seki, and H. Inokuchi, J. Chem. Phys. 77, 4870 (1982).

${ }^{28}$ (a) D. Haarer, Chem. Phys. Lett. 31, 192 (1975); (b) C. P. Keijzers and D. Haarer, ibid. 49, 24 (1977); (c) D. Haarer and M. Philpott, in Spectroscopy and Excitation Dynamics of Condensed Molecular Systems, edited by V. M. Agranovich and R. M. Hochstrasser (North-Holland, Amsterdam, 1983).

${ }^{29}$ N. Karl and J. Ziegler, Chem. Phys. Lett. 32, 438 (1975).

${ }^{30}$ E. C. M. Chen and W. E. Wentworth, J. Chem. Phys. 63, 3183 (1975).

${ }^{31}$ D. Haarer, J. Chem. Phys. 67, 4076 (1977), Fig. 10.

${ }^{32}$ W. Steudle, Doktorarbeit, Universität Stuttgart, 1979, Fig. 4.1.

${ }^{33}$ Obtained from the atomic coordinates, taken from the literature (Refs. 18 and 35), and from Table II, and an appropriate hydrogen van der Waals radius, $r_{w}=1.04$.

${ }^{34}$ Average tilt angle of the four independent molecules in the unit cell $(Z=6)$, calculated from the atomic coordinates of Ref. 4 , in agreement with the angles derived from the equations of the molecular planes of Ref. 4 , but in contradiction to the numerical values given in Ref. 4 .

${ }^{35}$ V. I. Ponomarev and G. V. Shilov, Sov. Phys. Crystallogr. 28, 397 (1983). 